\title{
Online Prediction of Milling Inner Hole Roundness Error Based on Accurate SSEM Value Extraction
}

\author{
Zhenbang Hu $\mathbb{D}^{1},{ }^{1,2,3}$ Gedong Jiang $\mathbb{D}^{1,2,3}$ Xuesong Mei $\mathbb{D}^{1,}{ }^{1,2,3}$ Xialun Yun $\mathbb{D}^{1,2,3}$ \\ and Yun Zhang (iD ${ }^{4}$ \\ ${ }^{1}$ State Key Laboratory for Manufacturing Systems Engineering, Xi'an Jiaotong University, 710048 Xi'an, Shaanxi, China \\ ${ }^{2}$ Shaanxi Key Laboratory of Intelligent Robots, Xi'an Jiaotong University, 710049 Xi'an, Shaanxi, China \\ ${ }^{3}$ School of Mechanical Engineering, Xi'an Jiaotong University, 710049 Xi'an, Shaanxi, China \\ ${ }^{4}$ School of Electro-Mechanical Engineering, Xidian University, 710071 Xi'an, Shaanxi, China
}

Correspondence should be addressed to Gedong Jiang; gdjiang@mail.xjtu.edu.cn

Received 2 February 2019; Accepted 10 July 2019; Published 5 August 2019

Academic Editor: Zhixiong Li

Copyright ( 92019 Zhenbang Hu et al. This is an open access article distributed under the Creative Commons Attribution License, which permits unrestricted use, distribution, and reproduction in any medium, provided the original work is properly cited.

To improve the machining accuracy and production efficiency of precision components with deep hole structures, an online prediction method of the inner hole roundness error, which cannot be directly measured in real time during the machining process, is proposed in this paper. For online prediction of the workpiece roundness error (WRE) during machining, a predictive model based on correlation analysis and a proportional method is proposed according to the spindle synchronous error motion (SSEM) by three-probe method testing. To improve the prediction accuracy of the WRE, a particle swarm optimization (PSO) algorithm is introduced for optimizing a probe mounting angle of a three-probe method, and a harmonic wavelet method for SSEM feature extraction is proposed. Using the PSO algorithm, the optimal probe mounting angle of the three-probe method is obtained, the influence of spindle surface roundness on SSEM is eliminated, and the higher-order harmonic suppression of the three-probe method is avoided effectively. By the harmonic wavelet method, the accurate SSEM extraction is enhanced and the WRE prediction accuracy is promoted. The experiments show that the inner hole roundness error online prediction method proposed in this paper has high prediction accuracy.

\section{Introduction}

Hydraulic cylinders are the key actuators in hydraulic systems and are widely used in the aerospace, automobile, and medical device industries. With further industrial developments, there is a tremendous 'demand for hydraulic cylinders in the manufacturing industry. This brings with it the challenge of high-accuracy machining and high-efficiency manufacturing of hydraulic cylinder components. Typically, an inner reciprocating cylinder moves along an outer cylinder, which is essentially a deep inner hole structure; therefore, high-precision machining is required to manufacture the outer cylinder. In particular, the surface roundness error of the inner hole is important due to its direct impact on the working performance and fault rates of hydraulic cylinders.
Traditional technology used for manufacturing inner holes is limited to drilling, boring, reaming, or broaching processes, which can be affected by the environment; moreover, precision is difficult to guarantee. Some advantages can be achieved by combining different technologies; however, there are still shortcomings, such as complicated processing methods and low efficiency. For high-precision machining of inner holes, various approaches have been presented and focus on improving the processing technology. Special tools have been integrated into various processing technologies designed as possible solutions. Zhang et al. studied on a customized gun drill regrinding system and the effect of apex offset variation on the hole straightness deviation though gun drilling experiments, and the research results show that the hole straightness deviation is the critical performance indicator in deep hole gun drilling process, and 
it is very difficult to achieve precise control for the gun drill tool geometry, so the customized drilling tool and the gun drill measurement system are hard to be widely popularized and applied [1]. Chin et al. investigated the effect of internally boring and trepanning association (BTA) drilling round profile, and they used the ratio of frequencies for modeling of deep hole geometry and the harmonic model for predicting the hole profile, but the method needs to use a special tool for BTA drilling, and the prediction model is unquantifiable processing [2]. Zhang et al. proposed a boring and trepanning association deep hole drilling mechanism, which uses a specialized staggered-edge drilling head with an asymmetric structure for machining holes with a large length-to-diameter ratio, and the influence of different factors on the functional behavior of deep hole machining and machining quality was investigated [3]. Aziz et al. developed a microcompound tool consisting of a drilling part and microdiamond-electroplated-grinding part to minimize burr formation and improve the surface roughness of the drilled holes [4]. However, developing special tools for different hole sizes results in excessive costs, and the methods often cannot be applied to other applications. High-speed milling (HSM) is an advanced processing technology that offers excellent efficiency and good quality and has gradually replaced surface grinding. HSM is a promising method as a half-finishing and finishing process for inner holes and can be used to machine inner holes of different sizes under different processing environments, with the potential to greatly improve hole machining efficiency. Nonetheless, surface roundness error of the inner hole remains a bottleneck to improving the precision of inner holes. Measuring and monitoring the surface roundness error during the milling of inner holes is therefore significant to improving the precision of HSM inner holes.

At present, surface roundness error measurements of inner holes are preformed offline, and milling efficiency is very low. Thus, studies of online methods to predict the inner hole roundness error are required. In milling, the spindle rotates relative to the average axis, and the radial error motion is defined as the relative displacement of the spindle from the average axis in the perpendicular direction on a certain axial plan. In general, the radial error motion is measured in a certain axial plane and includes both synchronous and asynchronous error motions. Synchronous error motion is an integer multiple of the rotational frequency, reflects the mean contour of the total error motion, and has good repeatability under certain conditions. During milling, the cutter rotates synchronously and repetitively with the spindle; therefore, the surface roundness error of the inner hole can be mapped by the spindle synchronous error motion. Ryua et al. used the character to flat end milling for generating the plane surface, and the surface texture characteristics are evaluated and predicted through a series of vector transformations considering tool run out and tool setting error [5]. Marsh et al. found that the workpiece diameter was affected by frequency content and phase of spindle, and they used simple grinding force models to predict best roundness of workpiece; the experimental results show the rate of roundness improvement matches theoretical predictions [6]. So the spindle error motion has a direct impact on WRE. With the spindle error motion measurement accuracy improvement, the predictive capability of workpiece roundness error will be enhanced.

The three-probe method is commonly used for spindle radial error motion measurements and is more appropriate for in situ measurements than conventional methods such as the Donaldson reversal method [7] and multiorientation method [8]. To accurately measure the error motion of the spindle using the three-probe method, the angles between the three measurement probes must be optimized and play a decisive role. The mounting angle of the probes determines whether the method is suitable for the required scenario, and a suitable angle combination could prevent the occurrence of higher-order harmonic suppression. Therefore, the influence of the probe mounting angles has widely been investigated. Obi et al. systematically demonstrated a sequential three-probe method and investigated the relevance of the measurement angle and coefficient of roundness, in addition to analyzing the characteristics and measurement errors of various angle combinations [9]. Similarly, Okuyama et al. demonstrated high-accuracy radial motion measurements using the three-probe method, along with a weighting equation and inverse filtering $[10,11]$. Shi et al. proposed a hybrid method to correctly estimate roundness error and considered the effects of stochastic disturbances using different measurement angles $\left(0^{\circ}, 42.8^{\circ}\right.$, and $126.4^{\circ}$ ) [12]. Moreover, Grejda implemented a unique adaptation of the Donaldson reversal technique to advance the state of spindle metrology using different angle combinations $\left(0^{\circ}, 122.5^{\circ}\right.$, and $\left.202.5^{\circ}\right)$ [13]. The methods are only applicable to the experimental environment, and the steps in these methods are cumbersome; in addition, the measurement accuracy needs to be further improved. In this paper, an optimization is performed to acquire the mounting angle of the probes, as well as the separation accuracy, and the performance of the novel angle combinations are compared to existing angle combinations.

The extraction of synchronous features of the measurement signal can have an important influence on predicting the inner hole roundness error. Synchronous feature extraction is centered on the characteristics associated with the integer multiples of the rotational frequency. Moreover, the synchronous feature extraction process can eliminate interference caused by noise in the signal. Many studies have focused on rotating machinery fault diagnosis using single signal analysis based on synchronous feature extraction. Siegel et al. used a tachometer-less synchronously averaged envelope method that uses signal processing and feature extraction techniques to diagnose the health and degradation of a rolling element bearings based on the synchronous characteristic signal [14]. Wang et al. performed signal feature extraction of rolling bearings with early weak faults: first, using the EEMD method to decompose the original signal into several IMFs; then, selecting the IMF with biggest kurtosis index value; and finally, using the tunable Q-factor wavelet transform for the fault signal feature extraction [15]. Synchronous feature extraction can also be used for signal purification. In this way, An et al. employed a mathematical 
morphology filter and combined the morphology filter with a simple algorithm, facile calculation, and effective filter to eliminate noise interference and purify the signal; nonetheless, the method was still affected by the characteristic of structural elements [16]. Zhang et al. combined synchronous feature selection and parameter optimization using an ant colony algorithm and support vector machine, to differentiate between different fault conditions, and demonstrated good final diagnostic accuracy for the intelligent fault diagnosis of rotating machinery [17]. Numerous methods have attempted feature extraction from characteristic signals. Chen et al. used the daubechies wavelet transform [18], and Lei et al. used the EEMD method [19]. However, most of these methods require complex algorithms; moreover, the harsh working conditions of many processing environments make it difficult to adopt these methods on-site for measuring rotating machinery error motion. Therefore, the harmonic wavelet method has been introduced to extract the synchronous features of measurement signals since it is suitable for in situ use and capable of accurate signal feature extraction, and importantly, can retain the basic characteristics of the measurement signal.

The online prediction method of the inner hole roundness error is needed for manufacturing inner holes as hydraulic cylinders. The method proposed in this paper has advantages of cost-effectiveness, high adaptability, and high accuracy. The work to improve prediction property mainly lies in the high-accuracy SSEM measuring and extracting method. The accurate predictive model is also essential. The rest of the paper is organized as follows: in Section 2, a novel method for the online prediction of the inner hole roundness error is introduced. In Section 3, the SSEM measurement and feature extraction method are presented, with a focus on improving measurement accuracy. Exactness of the SSEM values were calculated. In Section 4, a predictive model based on a correlation analysis and experimental data is presented. In Section 5, results of experimental validation are presented, and in Section 6, discussions on the various angle combinations are provided. Finally, the conclusions are summarized in Section 7.

\section{Method for WRE Online Prediction}

The high-speed spindle and cutting tool rotate together during the inner hole milling process; therefore, if the spindle and the cutting tool are ideally connected, their motions are synchronized and consistent. The internal surface of the workpiece is affected by cutting tool error motion; in particular, the workpiece roundness profile is highly influenced by the cutting tool error motion during hole milling. It follows that the error motion radius will lead to a corresponding roundness profile radius.

In the general case, the roundness profile of the machined internal surface is definite, owing to the repeatability of spindle radial error motion. The SSEM is extracted from the spindle radial error motion and plays a crucial role in this repeatability. This paper proposes a novel method for WRE online prediction using the accurate SSEM value along with the mathematical model. The procedure can be outlined as follows:

(1) Construct the experimental system for accurate spindle radial error motion measurement, which must satisfy the requirements for online measurements under certain machining conditions. Select a measurement method that can eliminate the interference factor and effectively improve measurement accuracy.

(2) Extract the spindle radial error motion signals to obtain the SSEM. Synchronous components of the signal have a much larger effect on WRE, and extracting the SSEM can effectively improve the signal-to-noise ratio (SNR) and prediction accuracy.

(3) Establish the predictive model using the extracted SSEM and workpiece roundness profile and determine whether there is a correlation between them.

(4) Predict the WRE according to the predictive models and experimentally validate the SSEM under the same machining conditions.

\section{Accurate Measurement of SSEM}

The proposed method aims to solve critical challenges that have an important influence on the final predicted result. One particularly important factor is the SSEM value. The first step in determining the SSEM is to acquire the spindle radial error motion. The key elements of this step are selecting a suitable measurement angle combination and extracting an accurate SSEM value.

3.1. Three-Probe Method for Measuring Spindle Radial Error Motion. The three-probe method is often used to calibrate the rotational accuracy of precision spindles and to acquire the surface roundness profile of an object; however, the method should not be limited to these applications. In this study, the three-probe method is used to measure spindle radial error motion and eliminate surface roundness profile. Consequently, the spindle radial error motion can be obtained with relatively high accuracy.

To perform this method, three probes are arranged at certain angles $\left(\theta_{1}, \theta_{2}, \theta_{3}\right)$ within the same cross section of a particular axial location. The intersection of the different directions is identified as the artifact origin $O$; moreover, $r_{\mathrm{L}}$ is the radius of the least square circle for measuring rotor surface.

The displacement probes measure the relative displacement in the sensitive direction between the probe and surface of the specified axial location of the rotor. The surface roundness profile can be expressed as $r(\theta)(\theta \in[0,2 \pi))$, and the displacement from the probe to the artifact origin can be defined as $R_{i}(i=1,2,3)$. Supposing the radial error motion of the spindle is comprised of the axis components $e_{x}(\theta)$ and $e_{y}(\theta)$ and considering the effects of the radial error motion in the sensitive direction, the signal measured by the displacement probe can be described by the following relational expression: 


$$
\begin{gathered}
s_{i}(\theta)=R_{i}-r_{\mathrm{L}}-r\left(\theta+\theta_{i}\right)-e_{x}(\theta) \cos \theta_{i}-e_{y}(\theta) \sin \theta_{i}, \\
(i=1,2,3 ; \theta \in[0,2 \pi)) .
\end{gathered}
$$

In general, one of the probe directions will overlap with the $x$-axis when $\theta_{1}=0^{\circ}$. The relationship between the radial error motion, surface roundness profile, and measurement signal can be expressed as

$$
\begin{aligned}
& e_{x}(\theta)=s_{1}(\theta)-r(\theta), \\
& e_{y}(\theta)=\frac{s_{2}(\theta)-r\left(\theta+\theta_{2}\right)-s_{1}(\theta) \cos \theta_{2}-r(\theta) \cos \theta_{2}}{\sin \theta_{2}} .
\end{aligned}
$$

From equations (2) and (3), the critical calculation of radial error motion can be obtained from the expression of $r(\theta)$, and in addition, the weighted combination process adopts the weighted coefficient $a_{i}(i=1,2,3)$, which transforms the measurement signal into a weighted combination function using $a_{1}=1, \quad a_{2}=\left(\sin \left(\theta_{3}\right)\right) /\left(\sin \left(\theta_{2}-\theta_{3}\right)\right)$, and $a_{3}=$ $\left(\sin \left(\theta_{2}\right)\right) /\left(\sin \left(\theta_{3}-\theta_{2}\right)\right)$.

At this point, the weighted combination function $s(\theta)$ only contains information about the surface roundness profile characteristics and demonstrates that $s(\theta)$ has periodicity; therefore, $S(\lambda)=\int_{-\infty}^{\infty} s(\theta) e^{-j \lambda \theta} d \theta$ is the frequency-domain expression of the weighted combination function of the measurement signal, $\lambda$ is the harmonic number, and the weighted function $F(\lambda)$ can be given as

$$
F(\lambda)=a_{1} e^{j \lambda 0}+a_{2} e^{j \lambda \theta_{2}}+a_{3} e^{j \lambda \theta_{3}} .
$$

According to equation (4), $r(\theta)$ can be derived using transposition and the inverse Fourier transform as

$$
r(\theta)=\frac{1}{2 \pi} \int_{-\infty}^{\infty}\left(\frac{S(\lambda)}{F(\lambda)}\right) e^{j \lambda \theta} d \lambda
$$

Thus, once the surface roundness profile of a specified axial location has been acquired from equation (5), by substituting equation (5) into equations (2) and (3), the radial error motion components $e_{x}(\theta)$ and $e_{y}(\theta)$ can be determined.

In addition, the three-probe method can be used to separate surface roundness profile and radial error motion from the measurement signals since it is also an error separation method; however, the measurement angle is the critical component in obtaining accurate separation results using the above analysis. Improper probe mounting angles will cause higher-order harmonic suppression to occur, and it will not be possible to obtain predetermined separation results from the measurements; thus, it is vital to select an appropriate set of measurement angles.

In the separation process, the weighted function $F(\lambda)$ and weighted combination function $S(\lambda)$ are crucial to obtaining the surface roundness profile $r(\theta)$, and the expression of the critical $F(\lambda)$ will directly influence future results.

\subsection{Enhancing the Measurement Precision}

3.2.1. Optimization of Defined Target. In the weighted function $F(\lambda)$, the measurement angle is the denominator, as shown in equation (5), such that a zero value of $F(\lambda)$ causes the method to lose significance; therefore, each value of the weighted function must be kept far from zero. First, it is necessary to ensure that each individual weighted value is kept far away from zero. If the minimum value of $F(\lambda)$ is far from zero, the other values must also be far from zero. Thus, if an arbitrary angle combination is given, as $\lambda$ changes, there must be a corresponding sequence of values, $F(\lambda)$. Find the minimum value, expressed as $\|F(\lambda)\|_{-\infty}$. The notation $\|\cdot\|_{-\infty}$ represents the minimum value of the numeric sequence. Since the angle combinations change, there will be many sequences $F(\lambda)$ and many minimum values from which the farthest from zero should be chosen. In other words, finding the maximum value can be expressed as \|\|$F(\lambda)\left\|_{-\infty}\right\|_{\infty}$, where the notation $\|\cdot\|_{\infty}$ represents the maximum value of the numeric sequence. Here, $A(\lambda)=\|\| F(\lambda)\left\|_{-\infty}\right\|_{\infty}$ is used to express the maximum value. Furthermore, the entire weighted function, i.e., the summation of the individual weighted values, should also be kept far away from zero and can be expressed as $B(\lambda)=\|\| F(\lambda)\left\|_{1}\right\|_{\infty}$, where the notation $\|\cdot\|_{1}$ represents the summation of all values.

Hence, the weighted function optimization is a multiobjective optimization, which incorporates the locality and global optimization goals, which are not independent and have mutual effects. To solve the multiobjective optimization problem, both the locality and global optimization goals must be considered. Then, the linear weighted combination method can be used to construct the combined optimization goals given as

$$
C(\lambda)=\alpha A(\lambda)+\beta B(\lambda)=\alpha\|\| F(\lambda)\left\|_{-\infty}\right\|_{\infty}+\beta\|\| F(\lambda)\left\|_{1}\right\|_{\infty},
$$

where $\alpha$ and $\beta$ are the weighting factors and must satisfy $\alpha+\beta=1$. The values of $\alpha$ and $\beta$ are determined based on the importance of the optimization goals. Generally, the locality optimization goals have a much greater influence on the separation accuracy and results for larger values of $\alpha$.

3.2.2. Optimized Angle Based on PSO. The PSO method is a multivariate and global optimization method with a number of advantages, including quick optimization, high efficiency, and reasonable accuracy, and thus has significance in many engineering applications.

The solution to the optimization problem corresponds to a particle in the PSO. The optimal searching process is used to first initialize a group of random particles, often referred to as the initial particle swarm, wherein each particle has a randomly generated initial value for its location and velocity. The particle swarm invariably follows the current optimal particle and iteratively updates the optimal particle to search for the optimal solution. During the iterative searching process, each particle is constantly updating its location and velocity within the search scope, and the updates during each 
iterative process are based on both the current optimal solution of the particle and the particle swarm. The current optimal solution of the particle is often referred to as the personal best $p_{\mathrm{b}}$, and analogously, the current optimal solution of the particle swarm is referred to as the global best $g_{\mathrm{b}}$. The location and velocity of the particle are updated according to

$$
\begin{aligned}
& v_{i j}^{k+1}=\sigma v_{i j}^{k}+c_{1} r_{1}\left(p_{\mathrm{bij}}^{k}-l_{i j}^{k}\right)+c_{2} r_{2}\left(g_{\mathrm{b} i j}^{k}-l_{i j}^{k}\right), \\
& l_{i j}^{k+1}=l_{i j}^{k}+v_{i j}^{k+1},
\end{aligned}
$$

where $i$ is the particle number; $j$ is the location or velocity dimension; $k$ is the number of iterations; $\sigma$ is the inertia weight; $l_{i j}^{k}$ and $v_{i j}^{k}$ are the location and velocity of the particle, respectively; $c_{1}$ and $c_{2}$ are the learning factors, usually $c_{1}=c_{2}=2$; and $r_{1}$ and $r_{2}$ are the random parameters in the range $[0,1]$. The method for obtaining the optimal solution of the angle combination based on the PSO search process is illustrated in Figure 1. The optimization procedure is as follows:

(1) Initialize the particle parameters and set the initial angle combinations and angular increments in advance

(2) Calculate the solution for the target function by using particle parameters and record them

(3) Obtain the local and global optimization targets based on the current particle parameters

(4) Update the particle parameters and identify the optimal solution

(5) Compare the current optimal value with the previous value and select the larger one

(6) Repeat steps (2) to (5) until the termination conditions are met

As shown in Figure 1, the measurement angles $\theta_{2}$ and $\theta_{3}$ are the search parameters, and the search range is $\left(0^{\circ}, 360^{\circ}\right)$. In applying the PSO method, the angle combination of $\left(0^{\circ}\right.$, $\left.\theta_{2}, \theta_{3}\right)$ refers to the particle's location $l_{i j}^{k}$, and the number of particles are randomly generated as $i$ such that a series of angle combinations $\left(0^{\circ}, \theta_{2 i}, \theta_{3 i}\right)$ can be acquired. In other words, the original particle swarm and angular increment $\left(0^{\circ}, d \theta_{2 i}, d \theta_{3 i}\right)$ are equivalent to the particle velocity $v_{i j}^{k}$.

In the optimal solution searching process, every particle location $l_{i j}^{k}$ corresponds to a serialized set of weighted functions $F(\lambda)$, and the corresponding value of $C(\lambda)$ can be obtained by continuously searching for the optimal solution, while updating the location and velocity of the particle according to equation (7). $p_{\mathrm{b} i j}^{k}$ is the particle location that fits the requirements of the locality optimized solution $A(\lambda)$, and $g_{\mathrm{b} i j}^{k}$ is the particle location that meets the requirements of the globally optimized solution for the combination of optimization goals $C(\lambda)$.

During the optimization process, as the current angle combination gets closer and closer to the optimal solution, the angular increments become smaller and smaller; therefore, the stopping criterion of the PSO is when the deviation of the angular increment is smaller than a predefined value or a preset number of iterations is reached.
Finally, using the PSO approach, the optimal measurement angle was acquired as $\left(0^{\circ}, 45^{\circ}, 96.5^{\circ}\right)$.

\subsection{Feature Extraction for Accurately Predicting SSEM.} The rotor of a high-speed spindle and cutting tool rotates together during the milling process; therefore, if error motion of the rotor and the cutting tool are ideally connected, they are synchronized and consistent. The milling workpiece internal surface is influenced by the cutting tool error motion, and in particular, the workpiece roundness profile is highly relevant to cutting tool error motion during hole milling. It follows that a larger error motion radius will lead to a larger roundness profile radius and vice versa.

In the general case, the roundness profile of the machined internal surface is repeatable due to the repetitive radial error motion. Therefore, the synchronous error motion of the cutting tool plays a particularly crucial role in determining the internal surface roundness profile. Hence, the synchronous error motion of the rotor could be used to predict the machined surface roundness, based on the feature extraction method, which extracts the synchronous error motion from the total error motion.

Thus, the accurate SSEM extraction problem can be transformed into a feature signal extraction problem. The harmonic wavelet was introduced to investigate the feature signal extraction of integer multiples of the rotational frequency since the characteristics of the extracted target frequency signal include strong background noise.

3.3.1. Accurate SSEM Extraction. The harmonic wavelet is a complex wavelet, first proposed by Newland [20], comprising a complex transformation of the time-domain and frequency-domain functions. The harmonic wavelet is suitable for extracting the signal of interest even when the signal is weak and difficult to acquire.

The harmonic wavelet transform can easily be realized in the frequency domain for practical engineering applications. First, Fourier transformation is applied to the signal. Then, it is transformed into the harmonic wavelet in the frequency domain. Finally, the inverse Fourier transform is used to reconstruct the signal in the time domain.

By investigating the feature signal extraction method, the ultimate aim is to acquire repeatable synchronous error motion $e_{s r}(\theta)$, which is comprised of the integer-harmonics signals, $e_{s x}(\theta)$ and $e_{s y}(\theta)$, extracted from the total error motions $e_{x}(\theta)$ and $e_{y}(\theta)$. Using the horizontal direction as an illustration, $e_{x}(\theta)$ is transformed into the frequency domain using Fourier transformation, and $H_{\text {exi }}\left(m_{i}, n_{i}, \lambda\right)$ is obtained from the harmonic wavelet, where $m_{i}, n_{i} \in R^{+}$, and $m_{i}<n_{i}$. Hence, one of the integer-harmonic signals can be acquired by performing an inverse Fourier transformation on $H_{\text {exi }}\left(m_{i}, n_{i}, \lambda\right)$, resulting in

$$
h_{\text {exi }}\left(m_{i}, n_{i}, \theta\right)=\frac{1}{2 \pi} \int_{-\infty}^{\infty}\left(H_{e x i}\left(m_{i}, n_{i}, \lambda\right)\right) e^{j \lambda \theta} d \lambda .
$$

According to the above approach, the sequential extraction of the integer-harmonic signals can be realized 


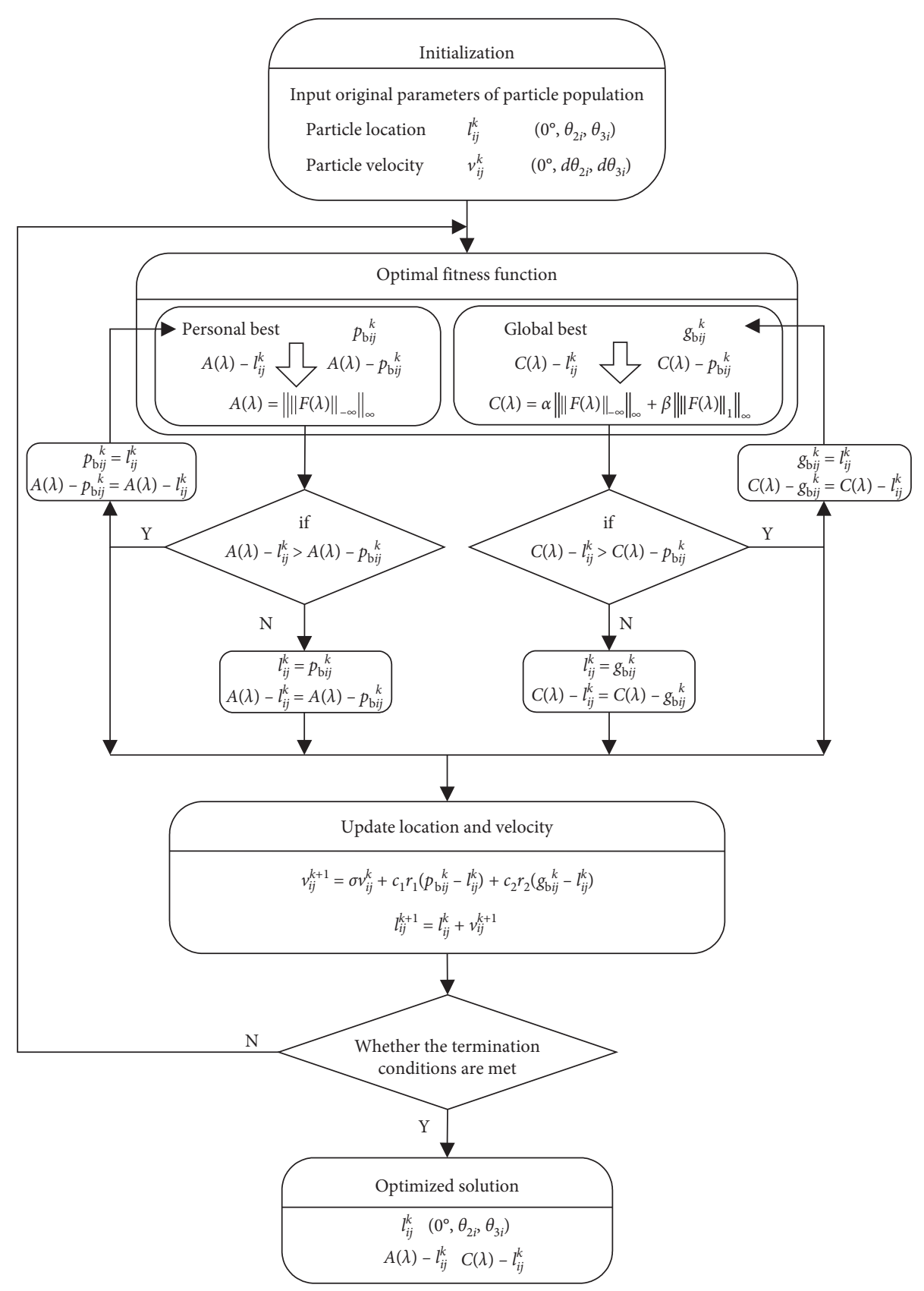

FIGURE 1: Process diagram for finding the optimal solution of the angle combination based on PSO.

according to the number of harmonics $i$ changed. The horizontal direction synchronous error motion $e_{s x}(\theta)$ is the superposition of all the integer-harmonic signals given as

$$
e_{s x}(\theta)=\sum_{i=1}^{N} h_{e x i}\left(m_{i}, n_{i}, \theta\right)
$$

Noise is extremely common in industrial engineering, and in particular, measurement signals acquired during the milling process usually contain significant noise components. Unfortunately, it is difficult to extract feature signals for a low SNR condition using conventional methods. In such cases, the harmonic wavelet is useful for performing feature signal extraction in the presence of strong background noise; hence, extracting both the exact amplitude and phase angle of integer-harmonics signals and fraction-harmonics signals is performed.

3.3.2. Calculation of SSEM Value. To verify whether the method is effective for feature extraction, spectrum analysis was performed on the measurement signal and extracted signal. The experiments were used to validate the accurate SSEM feature extraction and performed under idle conditions. The measurement signals acquired at a rotational speed of $4800 \mathrm{r} / \mathrm{min}$ were separated into the surface roundness profile and spindle radial error motion $e_{r}(\theta)$ using the three-probe method. Subsequently, SSEM $e_{s r}(\theta)$ 
was extracted from spindle radial error motion $e_{r}(\theta)$, and a frequency spectrum analysis was carried out. The spindle radial error motion and SSEM are illustrated in Figures 2 and 3 , respectively.

The SSEM demonstrates better repeatability in the polar plot and the integer-harmonics are more distinct in the frequency domain; moreover, the extracted signals are smoother, and the influence of noise is negligible in the time domain, as well as the frequency domain.

Hence, the harmonic wavelet meets the extraction requirements for spindle radial error motion, and the harmonic wavelet is suitable for feature signal extraction in the presence of strong background noise. In addition, the repeatability and periodicity of the total error motion can be determined.

The SSEM value is calculated as the difference between the radii of the maximum external circle $r_{\text {mec }}$ and minimum inner circle $r_{\text {mic }}$. The spindle radial error motion value was calculated at $4.3 \mu \mathrm{m}$, as shown in Figure 2, and the corresponding SSEM value was calculated at $3.58 \mu \mathrm{m}$, as shown in Figure 3 . The signals contain noise which will lead to lower accuracy since noisy signals expand the outer boundary and reduce the inner boundary, resulting in an inner minimum circle with a smaller radius and a maximum external circle with a larger radius. The difficulty degree for feature signal extraction in complex algorithms and the harsh working conditions is significantly reduced compared to previous approaches [16-19]. Thus, feature signal extraction can effectively improve the accuracy of the SSEM value, thereby improving the overall accuracy of the predictive model.

\section{Modeling with Correlation Analysis of SSEM and WRE}

4.1. Experimental Setup. The proposed method was validated experimentally and the relationship between WRE and SSEM values during the milling process were examined. The experiment was used to characterize the direct influence of the error motion and surface roundness error and to derive a mathematical relationship between them. Inner shallow holes were machined at different rotational speeds, and the spindle radial error motion under the milling process was acquired.

4.1.1. SSEM Measurement. Experiments were conducted using a vertical-type machine tool (BCH850, SFMTP). The experimental setup is shown in Figure 4. A multichannel synchronization signal acquisition system, developed by our group, was also used. The core component (USB-4432, NI corporation) has a 24 bit resolution and was used to acquire analog signals and convert them into digital signals. The system used three displacement sensing systems (NCDT3010-M-S1) and S1 eddy current probes (MicroEpsilon) with a range of $1 \mathrm{~mm}$, and a resolution of $0.05 \mu \mathrm{m}$ was used for obtaining measurements. A signal conditioner was used for signal conditioning and signal amplification, and a stand was used to hold the probes at the optimal angles $\left(0^{\circ}, 45^{\circ}, 96.5^{\circ}\right)$. The program for controlling the system was developed in LabVIEW, using the signal acquire, process, plot, and store functions.

The sampling frequency of the recorded displacement signals was $10 \mathrm{kHz}$, and the signal acquisition card ensured the signals were based on the different channel measurements from each probe, with synchronous sampling at evenly spaced angular increments. In other words, the number of sampled points per revolution remained consistent even as the rotational speed changed.

In the angular domain, every sampling point corresponded to an angle of uniform distribution; therefore, the spindle radial error motion, or more specifically, the accurate SSEM, was displayed as the relative position in the angular domain. Thus, the position of the accurate SSEM corresponds to the position of the cutting tool under the milling process in the angular domain, which means the accurate SSEM corresponds to the workpiece surface roundness profile in the angular domain.

4.1.2. Roundness Measurement to Determine WRE. The measurement signals of the probes distributed by different angles were acquired by the system at a stable rotational spindle speed during the milling process and separated into the spindle radial error motion using the three-probe method presented in Section 3.1. Then, feature extraction was performed to obtain the spindle radial error motion and SSEM.

The SSEM was obtained, and the corresponding workpiece surface roundness profile was measured using a roundness measuring instrument (YD200, LHCT company). In the measuring process, the machining workpiece first needed to be placed in the appropriate position. The inner hole roundness of machining workpiece measurement is shown in Figure 5.

4.2. Establishment of Predictive Model. The predictive model is based on actual measurements. Signals were acquired from a spindle working to cut the workpiece at a stable rotational speed of $4200 \mathrm{r} / \mathrm{min}$, and accurate SSEM values is shown in Figure 6(a). Measurements of the inner hole roundness of the machined workpiece is presented in Figure 6(b).

It can be seen from Figure 6 that the accurate SSEM has a similar shape to the workpiece roundness profile. To verify this, a similarity analysis of the image histograms was performed and to determine the degree of similarity. The histogram, usually referred to as a fingerprint image, depicts the intrinsic properties of the image and is not affected by size, position, or rotational angle.

4.2.1. Correlation of Measurement Results. To investigate morphological similarities between accurate SSEM and workpiece roundness profile, the plots were extracted, converted to grayscale images $(190 \times 200$ pixels), and histograms of the grayscale images were generated. As an example, histograms for a spindle rotational speed of 4200 r/min are shown in Figure 7. 

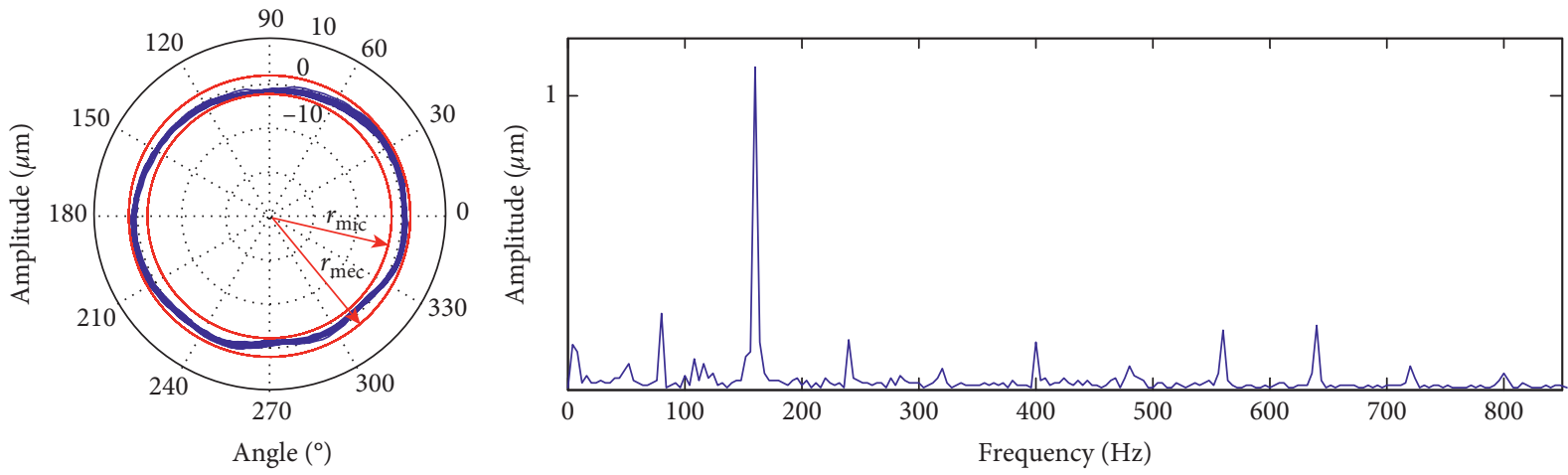

FIgURE 2: Spindle radial error motion separated from the measurement signal using the three-probe method.
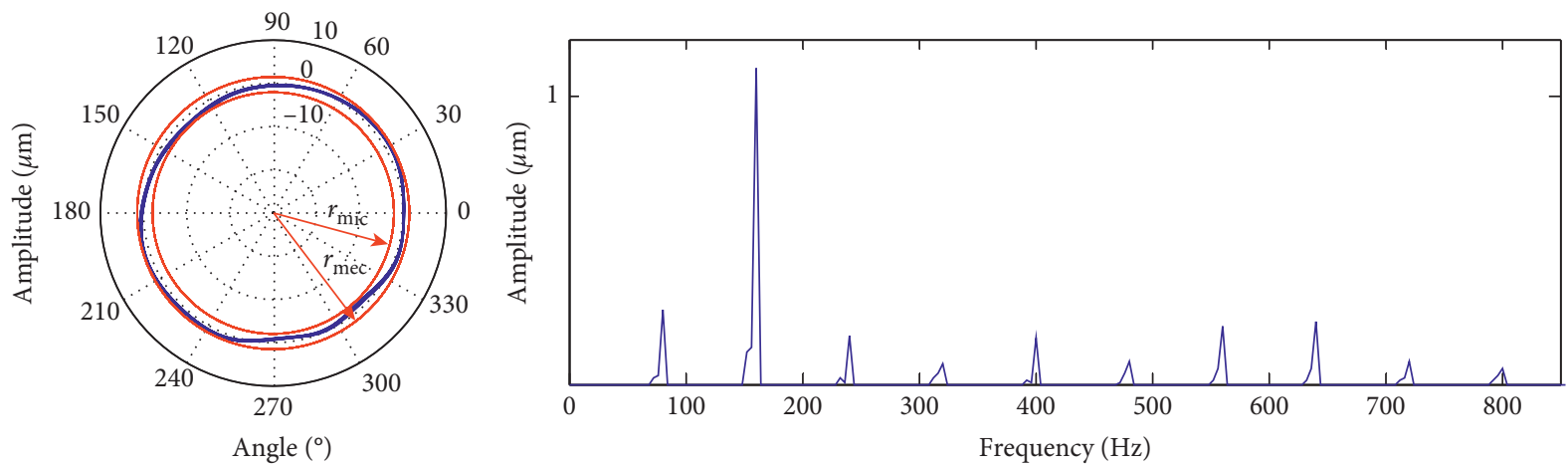

FIGURE 3: SSEM extracted from the spindle radial error motion using the harmonic wavelet.

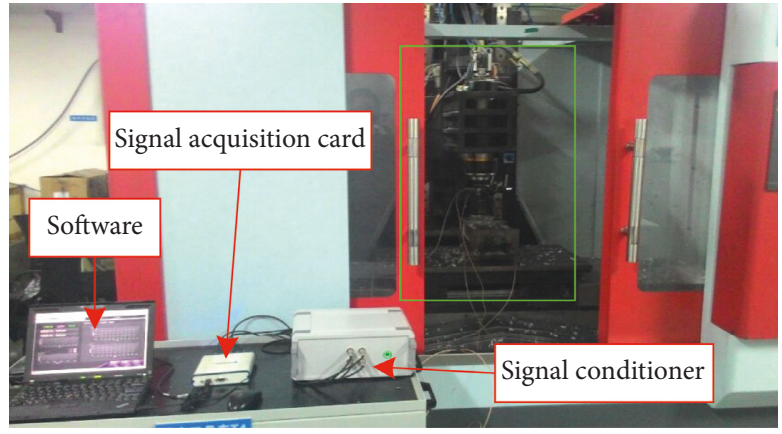

(a)

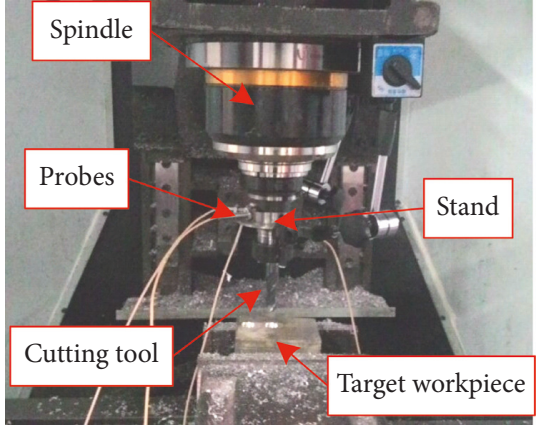

(b)

FIgURE 4: Experimental setup.

From Figure 7, it can be seen that the distributions of pixels amongst the grayscale values are close to each other in both histograms. The degree of similarity between histograms can be judged in many ways, including distance judgment methods commonly selected for comparing image morphologies. The histogram intersection distance and the distance proposed by Bhattacharyya et al. can indicate similarity between histograms and only differ in their expression.

The histogram intersection distance reflects the number of repeated pixels in the histogram, and the result can be normalized to be within the range $[0,1]$ by dividing the histogram intersection distance by the number of pixels in one of the histograms. Similarity between histograms is higher as the normalized result gets closer to 1 . The normalized histogram intersection distance $D_{\text {nhi }}$ can be calculated as

$$
D_{\mathrm{nhi}}=\frac{\sum_{i=1}^{N_{p}} \min \left(H_{\mathrm{GS}}(i), H_{\mathrm{GR}}(i)\right)}{\sum_{i=1}^{N p} H_{\mathrm{GS}}(i)},
$$

where $H_{\mathrm{GS}}(i)$ is the histogram of the accurate SSEM, $H_{\mathrm{GR}}(i)$ is the histogram of workpiece roundness profile, and $N_{p}$ is the total number of pixels. The normalized histogram intersection distance $D_{\text {nhi }}$ is 0.9989 .

Analogously, the Bhattacharyya distance reflects the probability distribution of the pixels of different histograms, 


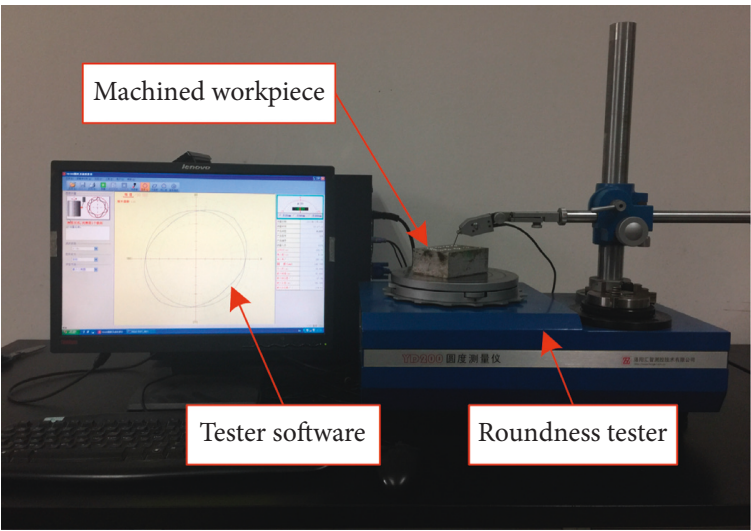

Figure 5: Roundness measurements using YD200.

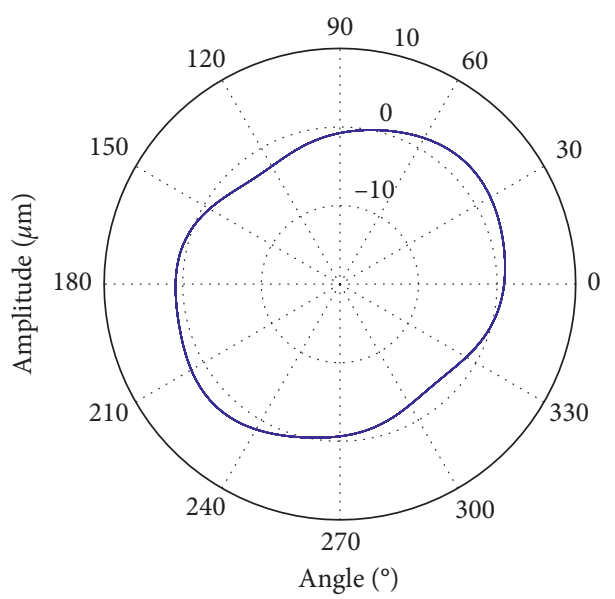

(a)

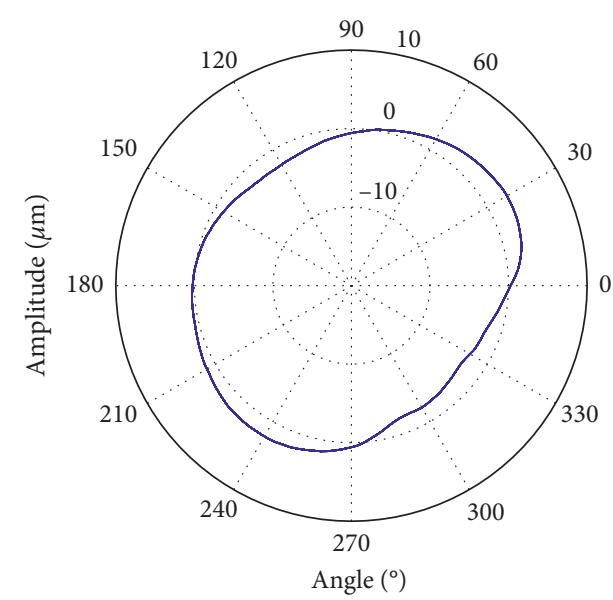

(b)

FIGURE 6: Measurements obtained during the machining process. (a) SSEM. (b) Workpiece roundness profile.

and its normalized function $D_{\mathrm{nB}}$ can be given as

$$
D_{\mathrm{nB}}=\frac{\sqrt{1-\sum_{i=1}^{N_{\mathrm{p}}} \sqrt{H_{\mathrm{GS}}(i) \cdot H_{\mathrm{GR}}(i)}}}{\sqrt{\sum_{i=1}^{N_{\mathrm{p}}} H_{\mathrm{GS}}(i) \cdot \sum_{i=1}^{N_{\mathrm{p}}} H_{\mathrm{GR}}(i)}} .
$$

In this case, the normalized histogram intersection distance $D_{\mathrm{nB}}$ is 0.0047 .

According to the distance judgment methods, the similarity between the accurate SSEM and workpiece roundness profile shows that the normalized histogram intersection distance is very close to 1 and the normalized Bhattacharyya distance is close to 0 . It follows that, under a steady rotational speed, both similarity judgment methods indicate that the histograms have a high degree of similarity in relation to image morphology.

4.2.2. Predictive Model. To establish the predictive model via numerical analysis, the relationship between the SSEM value and the WRE must be determined, as well as the relationship between data sequences of SSEM and workpiece roundness profile. The SSEM value and the WRE can be calculated from the SSEM and workpiece roundness profile, respectively. The
SSEM value, the WRE, and the ratio between them are presented in Table 1. A correlation analysis can be introduced to obtain the relationship between data sequences of the accurate SSEM and workpiece roundness profile. The correlation coefficient for the two correlated sequences can be defined as

$$
\rho_{\left(e_{s r}(\theta), r_{t w}(\theta)\right)}=\frac{\sum_{\theta}^{\theta_{\text {end }}}\left(e_{s r}(\theta)-\overline{e_{s r}(\theta)}\right) \cdot\left(r_{t w}(\theta)-\overline{r_{t w}(\theta)}\right)}{\sqrt{\sum_{\theta}^{\theta_{\text {end }}}\left(e_{s r}(\theta)-\overline{e_{s r}(\theta)}\right)^{2} \cdot \sum_{\theta}^{\theta_{\text {end }}}\left(r_{t w}(\theta)-\overline{r_{t w}(\theta)}\right)^{2}}} .
$$

The correlation coefficient for the SSEM value and WRE was acquired and is presented in Table 1.

As seen in Table 1, the SSEM value and WRE are directly proportional and the proportionality coefficient $K_{\mathrm{Ra}}$ is 0.88 . Assuming the SSEM value is known, the proportionality coefficient $R_{e s r}$ can be used to establish the predictive model, and WRE can be expressed as $R_{t w}=c_{p} R_{e s r}=\left(1 / K_{\mathrm{Ra}}\right)$. $R_{e s r}=1.14 R_{e s r}$. The proportional relationship between the SSEM value and WRE is a comprehensive technical indicator but only reflects the numerical relationship in a single dimension. This oversimplified model for online WRE prediction is not accurate enough. 


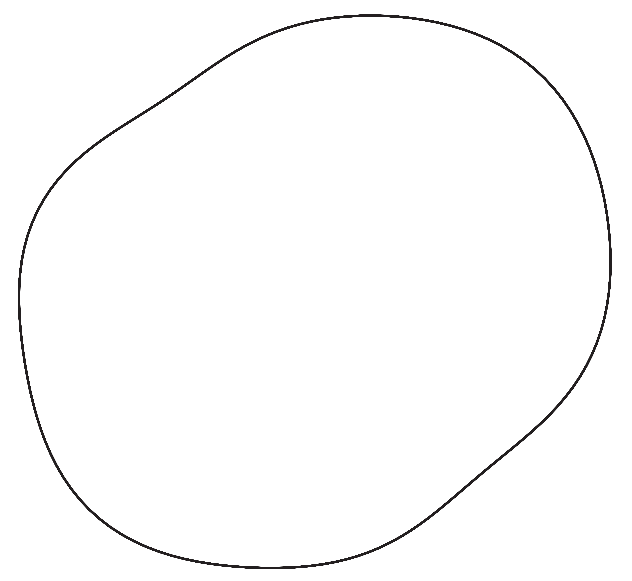

(a)

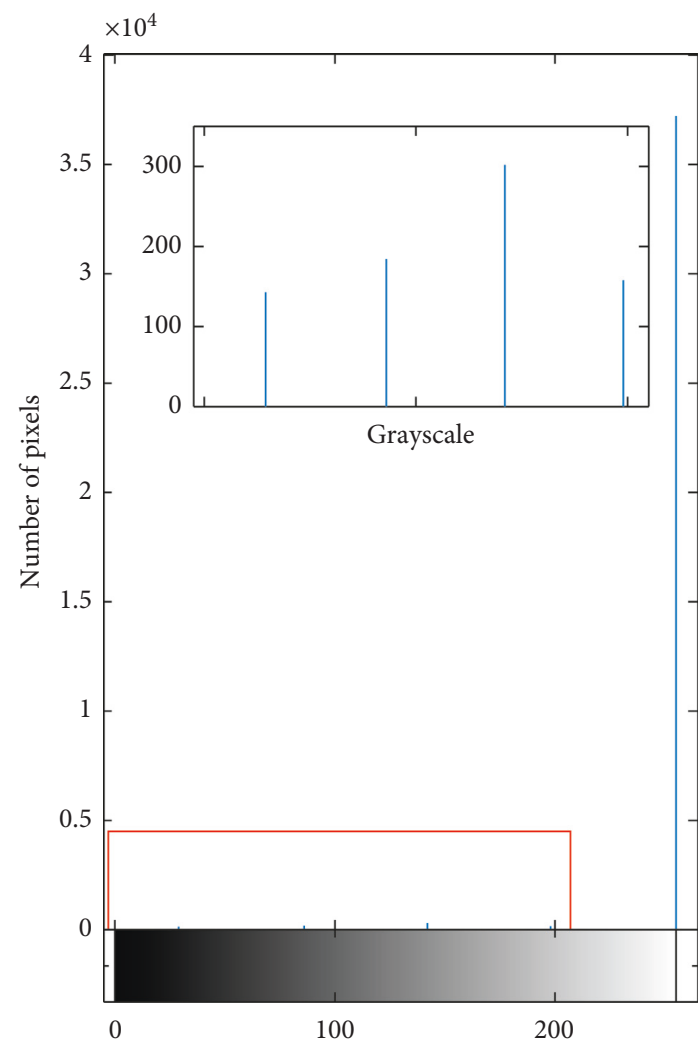

(c)

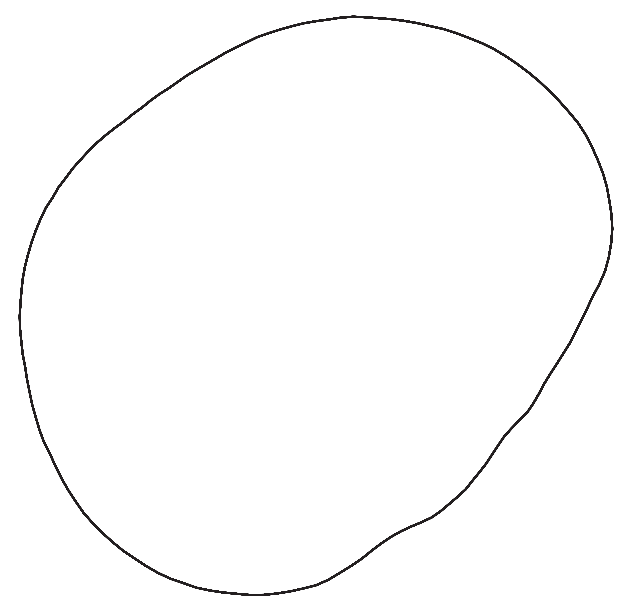

(b)

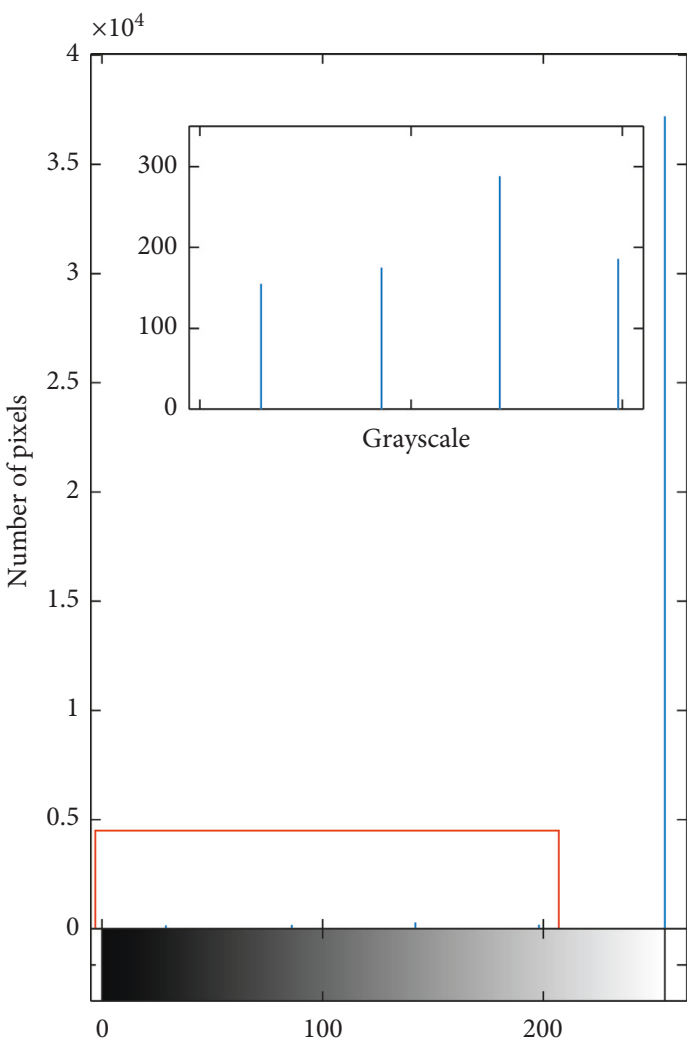

(d)

FIGURE 7: Initial images and histograms. Grayscale image of (a) accurate SSEM and (b) workpiece roundness profile. Histogram of (c) accurate SSEM and (d) workpiece roundness profile.

TABLE 1: Results of proportional analysis and correlation analysis.

\begin{tabular}{lcccc}
\hline Rotational speed $(\mathrm{r} / \mathrm{min})$ & SSEM value $R_{e s r}(\mu \mathrm{m})$ & WRE $R_{t w}(\mu \mathrm{m})$ & Ratio $K_{R a}=\left(R_{e s r} / R_{t w}\right)$ & Correlation coefficient $\rho_{\left(e_{s r}, r_{t w}\right)}$ \\
\hline 4200 & 5.04 & 5.75 & 0.88 & 0.91
\end{tabular}

Furthermore, the correlation coefficient $\rho_{\left(e_{s r}(\theta), r_{t w}(\theta)\right)}$ is 0.91 , which is very large and suggests the SSEM and workpiece roundness profile are highly correlated, according to the numerical analysis. The correlation coefficient consistently reflects trends in the signal sequences of the SSEM and the workpiece roundness profile more detailed, equivalent to extending the dimensions of the analysis, and can therefore be used as a correction factor in the predictive model along with proportionality coefficient for WRE, which comprehensively reflects the performance of the tool. 
Based on the proportionality coefficient and correction factor, the predictive model can be established as

$$
R_{t w}=c_{p c} R_{e s r}=\left(\frac{1}{K_{R a} \cdot \rho\left(e_{s r}(\theta), r_{t w}(\theta)\right)}\right) \cdot R_{e s r}=1.25 R_{e s r} .
$$

The necessary correlation between the SSEM value and WRE was found to exist, and the relationship can be defined using a precise mathematical formula, which can then be incorporated into the predictive model. At the same time, based on proportionality and the correlation analyses, the predictive model can be obtained. Both the proportionality coefficient and the correlation coefficient play an important role in the predictive model.

According to the description above, when the inner surface quality of the machined workpiece is difficult to determine by direct measurement during the machining process, the WRE is the most concerned parameter in deep hole machining and can be indirectly predicted using the online prediction method. For SSEM and workpiece roundness profile measurements exhibiting high morphological similarity, a correlation analysis was performed. Based on the results, the predictive model was established and used for online prediction of the inner hole roundness error. Thus, the method can improve upon existing online monitoring techniques and enhance machining accuracy.

\section{Results and Discussion}

5.1. Experimental Validation of Predictive Model. To validate the inner hole roundness error online prediction method, inner holes were machined by a continuous milling process using the parameters of the model. The SSEM was measured online at the rotational speed of $4200 \mathrm{r} / \mathrm{min}$ by the experimental setup mentioned in Section 4.1, and the values were obtained using the method described in Section 3.3 and are presented in Figure 8.

From Figure 8, it can clearly be observed that SSEM is irregular in morphology. The calculated SSEM values and predicted results of the different models are presented in Table 2, including the model using only the proportionality coefficient as well as the corrected model.

To verify exactness of the predicted results, the workpiece surface roundness profiles were tested by the roundness measuring instrument mentioned in Section 4.1 and shown in Figure 9. Numerical characteristics of workpiece surface roundness profiles are listed in Table 2, and a comparative analysis of the results is also provided.

Figure 9 shows that similarity also exists between the SSEM and workpiece surface roundness profile. The comparative analysis of the numerical characteristics provides accurate and intuitive data, as shown in Table 2. Advantages of the different models were compared by extracting the absolute value of the deviation and percentage differences. In the composite model, deviations from the predicted results can be controlled within $0.5 \mu \mathrm{m}$ (a difference of less than 7\%) and the prediction accuracy is significantly improved compared to results of the naive model, with deviations reaching $1.27 \mu \mathrm{m}$ (a difference of 11.09\%). Comparing with research results of Ryua et al. [5] and Marsh et al. [6], the predicted results here not only give the qualitative analysis results but also provide the quantitative analysis results, and it has high accuracy. Thus, it can be concluded that the method proposed in this paper could be used to accurately predict WRE online.

\section{Discussion}

The combination of probe angles for measuring SSEM has a large effect on the weighted function $F(\lambda)$. Herein, we discuss how certain angle combinations affect the performance of $F(\lambda)$ as well as characteristics of the optimized angle combination proposed in this paper.

The fundamental harmonic of the measurement signal $(\lambda=1)$ is related to eccentric mounting (rotor eccentricity), and the effects of rotor eccentricity can be eliminated by using eccentricity compensation. The 2 nd to 50 th harmonics of the measurement signal $(2 \leq \lambda \leq 50)$ make up the surface roundness profile. Higher frequency harmonics $(\lambda>50)$ are usually presented as surface waviness or roughness. Therefore, in analyzing the surface roundness profile, a performance assessment of the weighted function $F(\lambda)$ needs to only consider the range $2 \leq \lambda \leq 50$.

The optimal angle actually corresponds to the value of $F(\lambda)$, according to every $\omega$ kept away from zero. The measurement angle using the conventional angle combination without optimization corresponding to $|F(\lambda)|$ is shown graphically in Figure 10.

As seen in Figure 10, the conventional measurement angle combination without optimization shows many values of $|F(\lambda)|$ satisfying the usage requirements; however, many values of the harmonic order $\lambda$ lead values of $|F(\lambda)|$ quite close to zero, and zero values of $|F(\lambda)|$ lead to harmonic suppression. Due to the harmonic suppression, the results for the surface roundness profile which are separated from the measurement signal using the threeprobe method will therefore be incorrect; moreover, an accurate measurement of the spindle radial error motion cannot be obtained.

For further verification, the angle combination acquired using the PSO was compared with the angle combinations proposed by Shi et al. $\left(0^{\circ}, 42.8^{\circ}, 126.4^{\circ}\right)[12]$ and Grejda $\left(0^{\circ}\right.$, $\left.122.5^{\circ}, 202.5^{\circ}\right)$ [13]. Then, $|F(\lambda)|$ was determined by substituting the measurement angle combinations into equation (4), as illustrated in Figure 11.

The results suggest that by using the optimized angle combination $\left(0^{\circ}, 45^{\circ}, 96.5^{\circ}\right)$, the minimum value of the weighted function $|F(\lambda)|$ is furthest from zero, as shown in Figure 11. Moreover, the entire weighted function appears to be further away from zero compared to functions associated with other angle combinations. Additional analyses of the results are necessary to verify this. The performance of different angle combinations were assessed based on several indexes including the minimum value $F_{\min }=\min \{|F(\lambda)|\}$, mean value $\mu$, difference between the mean value and standard deviation $\delta=\mu-s_{\mathrm{d}}$, 


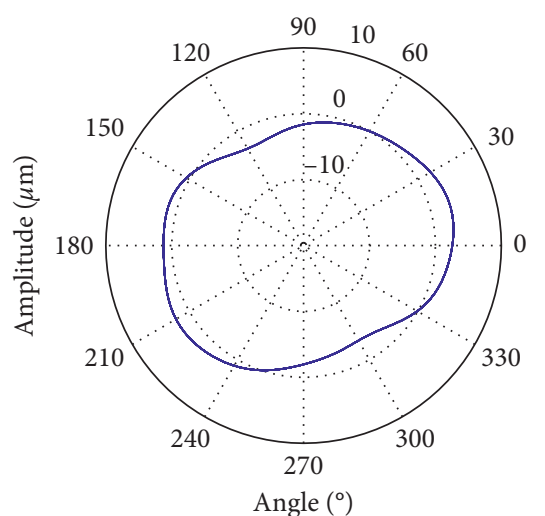

(a)

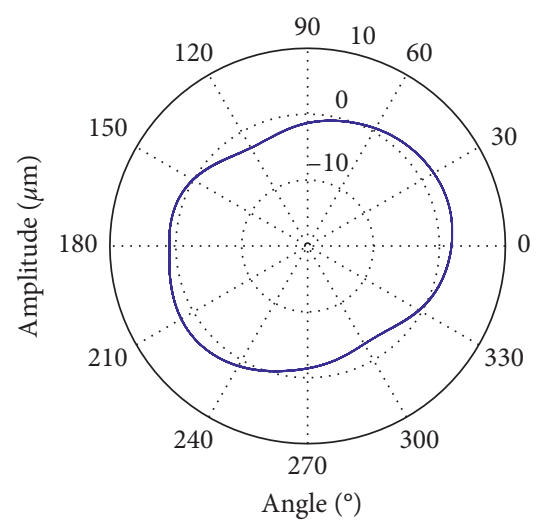

(c)

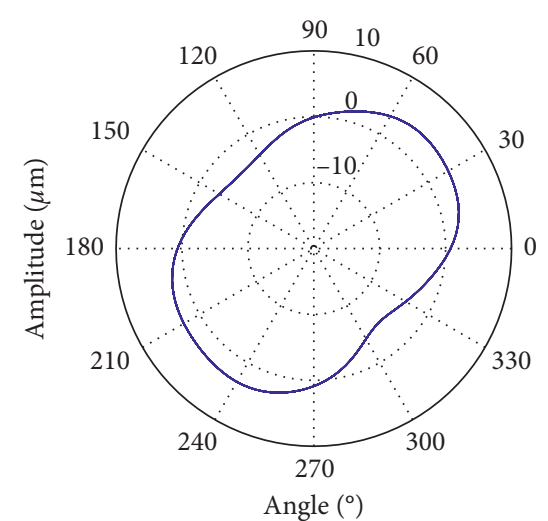

(b)

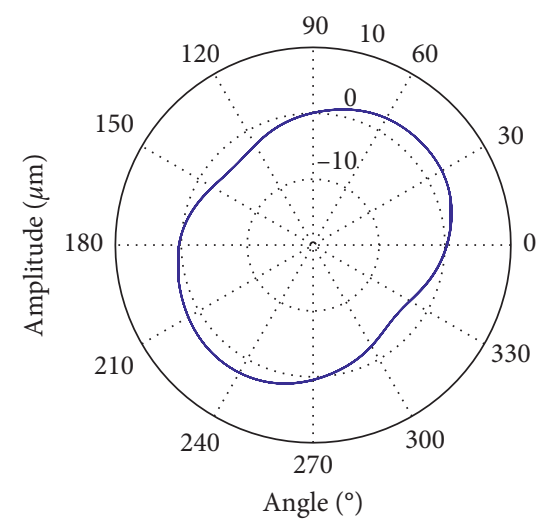

(d)

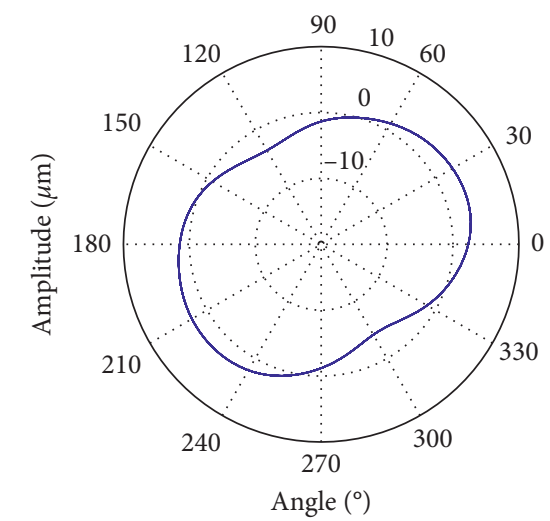

(e)

FIGURE 8: Online measurements of SSEM for experimental validation of the predictive model.

and the number of values close to zero $n_{\mathrm{cz}}(|F(\lambda)|<0.2)$, as shown in Table 3.

The conventional measurement angle combinations tend to be close to zero at the minimum value $F_{\min }$, which results in harmonic suppression. In comparison, the optimized angle combinations push $F_{\text {min }}$ further away from zero, and as the minimum value becomes larger, the harmonics become unsuppressed. Analogously, the number of values close to zero should be as small as possible and is an important index to invalidate the error separation method. As an initial criterion, each individual index can be used to screen for the most suitable angle combinations.
In contrast to the individual index, for situations in which the minimum value is not close to zero, however, the individual weighted value is close to the minimum, i.e., the entire weighted function is close to zero, so there is a higher probability of harmonic suppression. Therefore, it is necessary to introduce global indexes to identify the angle combinations with superior performance. The mean value $\mu$ and the difference between the mean value and standard deviation $\delta$ are global indexes that can be used to ensure the entire weighted function is kept far from zero.

Of particular importance is that there is some interaction between individual and global indexes, which means that for 
TABle 2: Predictive performance analysis.

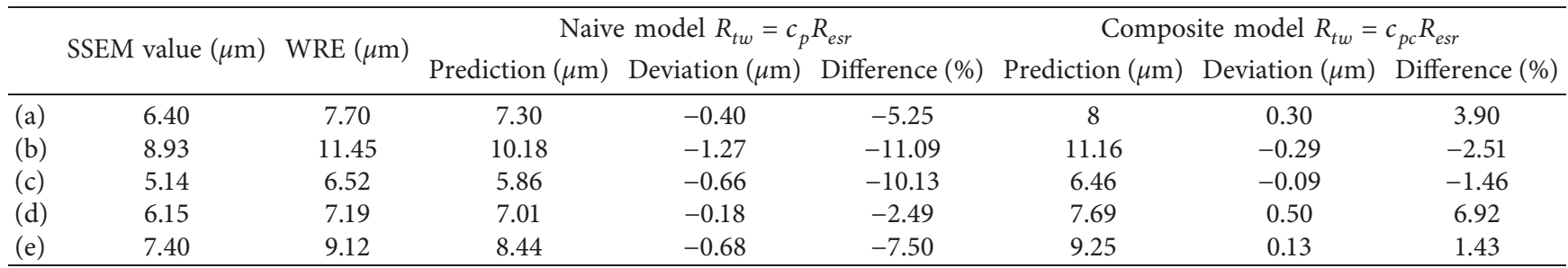

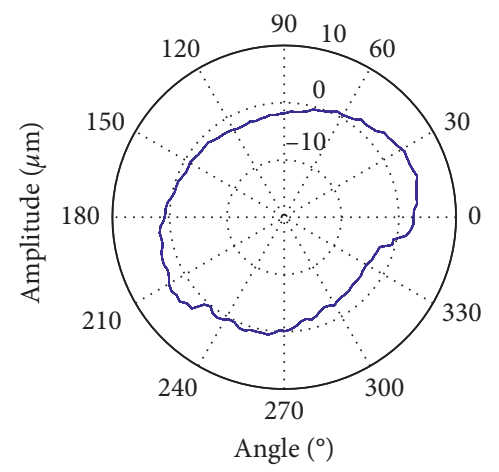

(a)

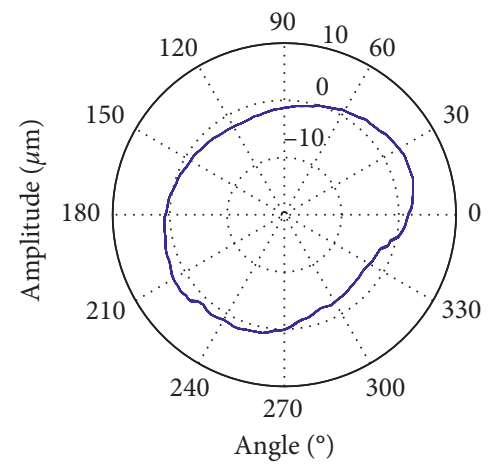

(c)

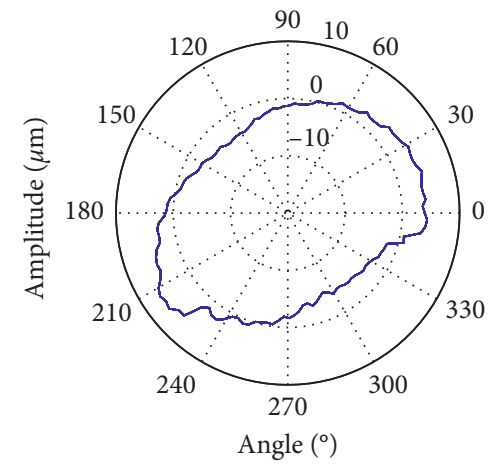

(b)

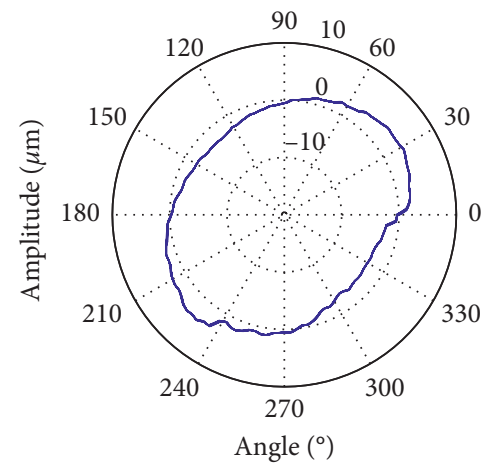

(d)

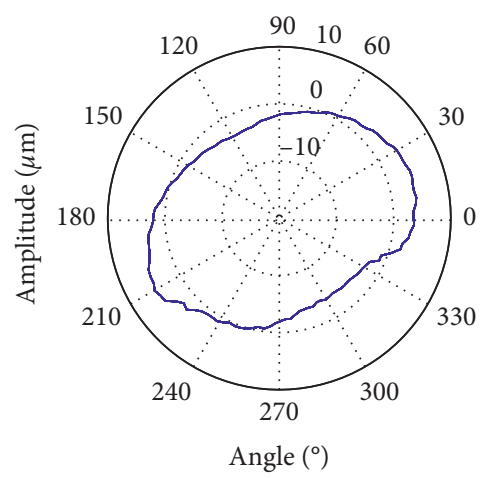

(e)

FIGURE 9: Workpiece surface roundness profile for verifying exactness of the predicted solutions.

larger $\mu$ values, $F_{\min }$ may be equal to zero and $n_{\mathrm{cz}}$ will be large. Examples of conventional measurement angle combinations are shown in Figure 10; however, these angle combinations are not suitable for high-accuracy measurement of the spindle radial error motion. This highlights the importance of the global index $\delta$ since the standard deviation represents volatility of weighted function and $\delta$ can be used to determine whether the entire weighted function with eliminated volatility is kept far away from zero. Taking the angle combination of $\left(0^{\circ}, 60^{\circ}, 90^{\circ}\right)$ as an example, $\mu$ is larger 


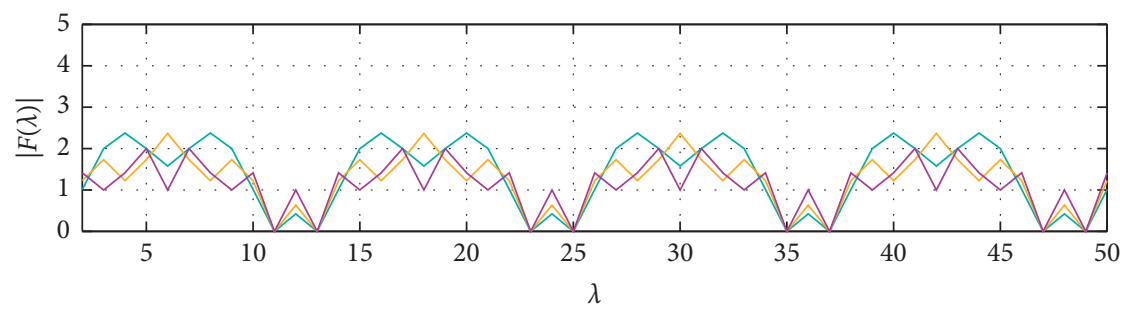

$\left(0^{\circ}, 30^{\circ}, 90^{\circ}\right)$

$-\left(0^{\circ}, 30^{\circ}, 120^{\circ}\right)$

$-\left(0^{\circ}, 30^{\circ}, 150^{\circ}\right)$

(a)

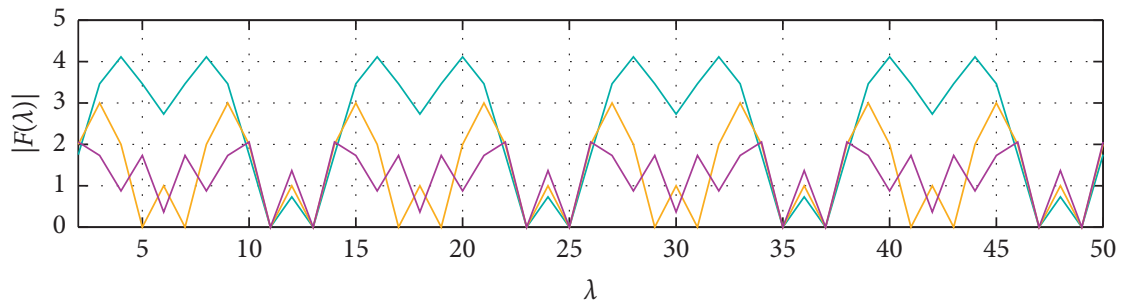

$-\left(0^{\circ}, 60^{\circ}, 90^{\circ}\right)$

$-\left(0^{\circ}, 60^{\circ}, 120^{\circ}\right)$

$-\left(0^{\circ}, 60^{\circ}, 150^{\circ}\right)$

(b)

FIGURE 10: Impact of the measurement angle combination on the weighted function $|F(\lambda)|$.

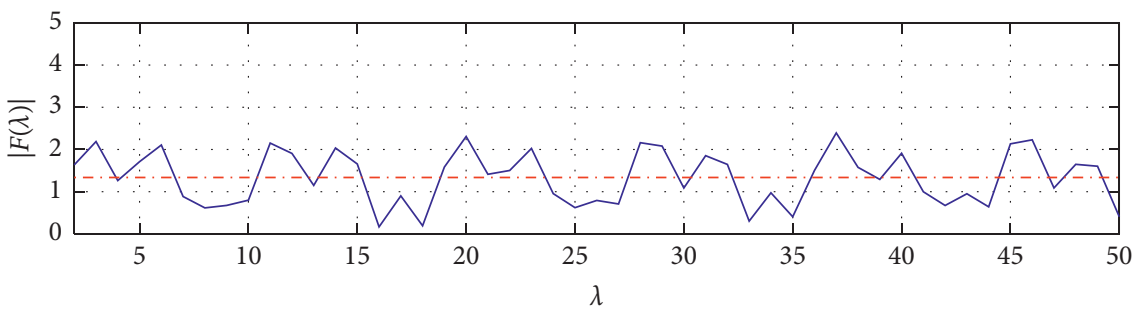

- $\left(0,42.8^{\circ}, 126.4^{\circ}\right)$

. . - Mean value

(a)

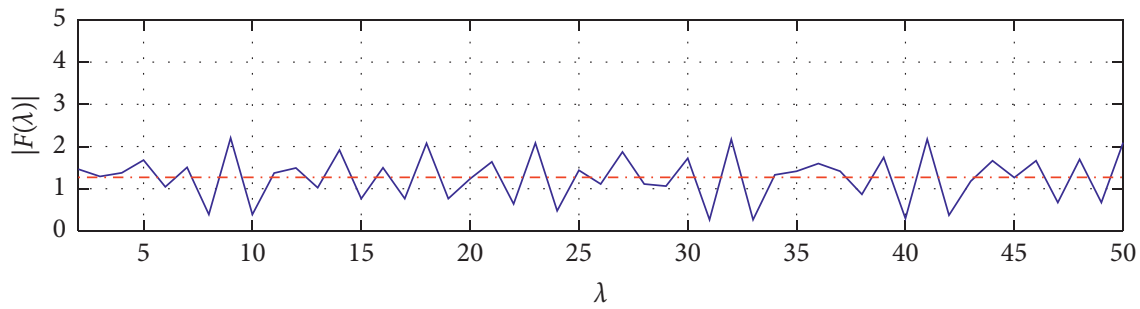

$-\left(0,122.5^{\circ}, 202.5^{\circ}\right)$

... Mean value

(b)

Figure 11: Continued. 


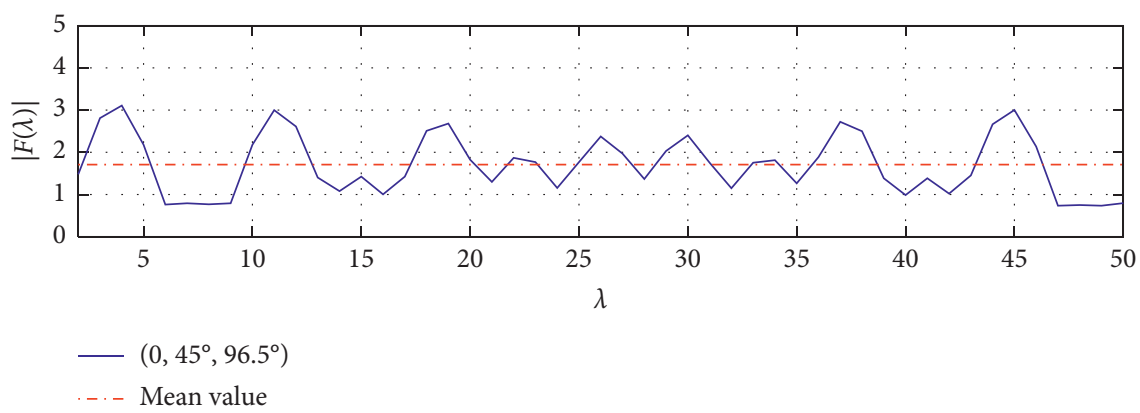

(c)

Figure 11: Comparison of the performance of different optimization measurement angle combinations.

TABle 3: Influence of different measurement angles on various indexes.

\begin{tabular}{lcccc}
\hline Measurement angle & $F_{\min }$ & $\mu$ & $\delta=\mu-s_{\mathrm{d}}$ & $n_{\mathrm{cz}}$ \\
\hline$\left(0^{\circ}, 30^{\circ}, 90^{\circ}\right)$ & 0 & 1.39 & 0.67 & 8 \\
$\left(0^{\circ}, 30^{\circ}, 120^{\circ}\right)$ & 0 & 1.24 & 0.76 & 8 \\
$\left(0^{\circ}, 30^{\circ}, 150^{\circ}\right)$ & 0 & 1.14 & 0.77 & 8 \\
$\left(0^{\circ}, 60^{\circ}, 90^{\circ}\right)$ & 0 & 2.41 & 0.26 & 8 \\
$\left(0^{\circ}, 60^{\circ}, 120^{\circ}\right)$ & 0 & 1.35 & 0.12 & 16 \\
$\left(0^{\circ}, 60^{\circ}, 150^{\circ}\right)$ & 0 & 1.23 & 0.68 & 8 \\
$\left(0^{\circ}, 42.8^{\circ}, 126.4^{\circ}\right)$ & 0.17 & 1.34 & 0.93 & 1 \\
$\left(0^{\circ}, 122.5^{\circ}, 202.5^{\circ}\right)$ & 0.27 & 1.27 & 0.96 & 0 \\
$\left(0^{\circ}, 45^{\circ}, 96.5^{\circ}\right)$ & 0.74 & 1.71 & 1.21 & 0 \\
\hline
\end{tabular}

compared to the values of other combinations, but $\delta$ is smaller. This suggests the value of $|F(\lambda)|$ has a large amount of volatility, the individual index $F_{\min }$ is equal zero, and $n_{\mathrm{cz}}$ is equal to 8 . Thus, the angle combination is not suitable. Both the individual and global indexes should comprehensively be considered when screening for the most appropriate angle combination.

Based on the results, the measurement angle combination of $\left(0^{\circ}, 45^{\circ}, 96.5^{\circ}\right)$, which was optimized using PSO, offers superior performance, as shown in Table 3 and Figure 11 . Therefore, the optimal angle of $\left(0^{\circ}, 45^{\circ}, 96.5^{\circ}\right)$ should be used to achieve high accuracy during the milling process and to investigate the spindle radial error motion of a highspeed spindle.

\section{Conclusion}

This paper proposed a novel method for online prediction of WRE based on the SSEM value, and mainly in better performance by comparison with previous studies. Results of the analysis show the method is feasible and practical. The main findings can be summarized as follows:

(1) The manufacturing accuracy of deep inner hole structure as the hydraulic cylinder is restricted to online measurement of machining quality; to solve the problem, the method based on accurate SSEM value extraction is proposed to predicted WRE during machining process, the concrete implementation processes, and technical essentials of this method are introduced in this paper, and this method has important academic meaning and value of practical application on high-accuracy manufacturing of deep inner hole.

(2) During high-speed hole milling, the accurate spindle radial error motion can be measured in real time using the three-probe method. The influence of surface roundness error was eliminated thereby improving measurement accuracy. The PSO algorithm was used to optimize the mounting angles of the probes according to the set of optimization goals. The optimized angle combination $\left(0^{\circ}, 45^{\circ}, 96.5^{\circ}\right)$ prevented higher-order harmonic suppression and enhanced measurement accuracy.

(3) The SSEM can be extracted from the spindle radial error motion with high accuracy using the harmonic wavelet even in the presence of strong background noise. Feature signal extraction of integer multiples of the rotational frequency provides the necessary analytical foundation for subsequent accurate calculations of the SSEM value.

(4) The predictive model established using the proportionality coefficient and correlation coefficient has high prediction accuracy, and deviations from true values can be controlled to be within an extremely small range.

Hence, the novel method could be used for online monitoring of machining quality during high-precision hole processing. Furthermore, the machining state of the machine tool can be adjusted online based on the predicted results, the control decision reference for dynamic balance can be provided online, and processing accidents can be avoided. Thus, the method offers the potential to drive high processing efficiency, advanced processing technologies, and good processing quality.

\section{Data Availability}

The data used to support the findings of this study are available from the corresponding author upon request.

\section{Conflicts of Interest}

The authors declare that they have no conflicts of interest. 


\section{Acknowledgments}

The authors gratefully acknowledge the support of 2018 National Development and Reform Commission "Internet+" Major Project Fund.

\section{References}

[1] X. Zhang, G. L. Tnay, K. Liu, and A. S. Kumar, "Effect of apex offset inconsistency on hole straightness deviation in deep hole gun drilling of inconel 718," International Journal of Machine Tools and Manufacture, vol. 125, pp. 123-132, 2018.

[2] D. H. Chin, M. C. Yoon, and S. B. Sim, "Roundness modeling in BTA deep hole drilling," Precision Engineering, vol. 29, no. 2, pp. 176-188, 2005.

[3] H. Zhang, X. Shen, A. Bo, Y. Li, H. Zhan, and Y. Gu, "A multiscale evaluation of the surface integrity in boring trepanning association deep hole drilling," International Journal of Machine Tools and Manufacture, vol. 123, pp. 48-56, 2017.

[4] M. Aziz, O. Ohnishi, and H. Onikura, "Innovative micro hole machining with minimum burr formation by the use of newly developed micro compound tool," Journal of Manufacturing Processes, vol. 14, no. 3, pp. 224-232, 2012.

[5] S. H. Ryua, D. K. Choi, and C. N. Chu, "Roughness and texture generation on end milled surfaces," International Journal of Machine Tools and Manufacture, vol. 46, no. 3-4, pp. 404-412, 2006.

[6] E. R. Marsh, A. W. Moerlein, T. R. S. Deakyne, and M. J. Van Doren, "In-process measurement of form error and force in cylindrical-plunge grinding," Precision Engineering, vol. 32, no. 4, pp. 348-352, 2008.

[7] R. R. Donaldson, "A simple method for separating spindle error from test ball roundness error," CIRP Annals-Manufacturing Technology, vol. 21, no. 1, pp. 125-126, 1972.

[8] K. Kim, "Development of microprocessor-based spindle error motion measurement system," Journal of Microcomputer Applications, vol. 8, no. 1, pp. 10-14, 1985.

[9] M. Obi, T. Kobayashi, and S. Furukawa, "On a new method of roundness measurement based on the sequential-three-points method," Transactions of the Japan Society of Mechanical Engineers Series C, vol. 54, no. 506, pp. 2475-2480, 1988.

[10] E. Okuyama, "Roundness measurement and the radial motion measurement based on the 3-point method using the inverse filtering," Proceedings of SPIE-the International Society for Optical Engineering, vol. 4222, no. 8, pp. 427-431, 2000.

[11] E. Okuyama and H. Moritoki, "A study on the roundness measurement and the radial motion measurement based on the 3-point method," Journal of Japan Society for Precision Engineering, vol. 65, no. 9, pp. 1312-1316, 1999.

[12] S. Shi, J. Lin, X. Wang, and M. Zhao, "A hybrid three-probe method for measuring the roundness error and the spindle error," Precision Engineering, vol. 45, pp. 403-413, 2016.

[13] R. D. Grejda, "Use and calibration of ultraprecision axes of rotation with nanometer level metrology," Doctor's Thesis, Pennsylvania State University, State College, PA, USA, 2002.

[14] D. Siegel, H. Al-Atat, V. Shauche, L. Liao, J. Snyder, and J. Lee, "Novel method for rolling element bearing health assessmenta tachometer-less synchronously averaged envelope feature extraction technique," Mechanical Systems and Signal Processing, vol. 29, no. 5, pp. 362-376, 2012.

[15] H. Wang, J. Chen, and G. Dong, "Feature extraction of rolling bearing's early weak fault based on EEMD and tunable Q-factor wavelet transform," Mechanical Systems and Signal Processing, vol. 48, no. 1-2, pp. 103-119, 2014.
[16] L. An, A. Hu, G. Tang, and L. Xiang, "Purification of rotor center's orbit with mathematical morphology filters," Chinese Journal of Power Engineering, vol. 25, no. 4, pp. 550-553, 2005.

[17] X. Zhang, W. Chen, B. Wang, and X. Chen, "Intelligent fault diagnosis of rotating machinery using support vector machine with ant colony algorithm for synchronous feature selection and parameter optimization," Neurocomputing, vol. 167, pp. 260-279, 2015.

[18] D. Chen, J. Fan, and F. Zhang, "An identification method for spindle rotation error of a diamond turning machine based on the wavelet transform," International Journal of Advanced Manufacturing Technology, vol. 63, no. 5-8, pp. 457-464, 2012.

[19] Y. Lei, Z. He, and Y. Zi, "Application of the EEMD method to rotor fault diagnosis of rotating machinery," Mechanical Systems and Signal Processing, vol. 23, no. 4, pp. 1327-1338, 2009.

[20] D. E. Newland, "Harmonic wavelet analysis," Proceedings of the Royal Society A: Mathematical, Physical and Engineering Sciences, vol. 443, no. 1917, pp. 203-205, 1993. 


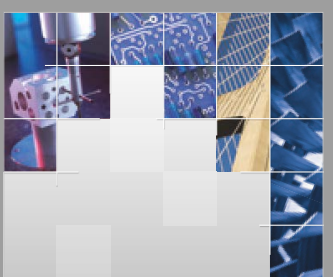

\section{Enfincering}
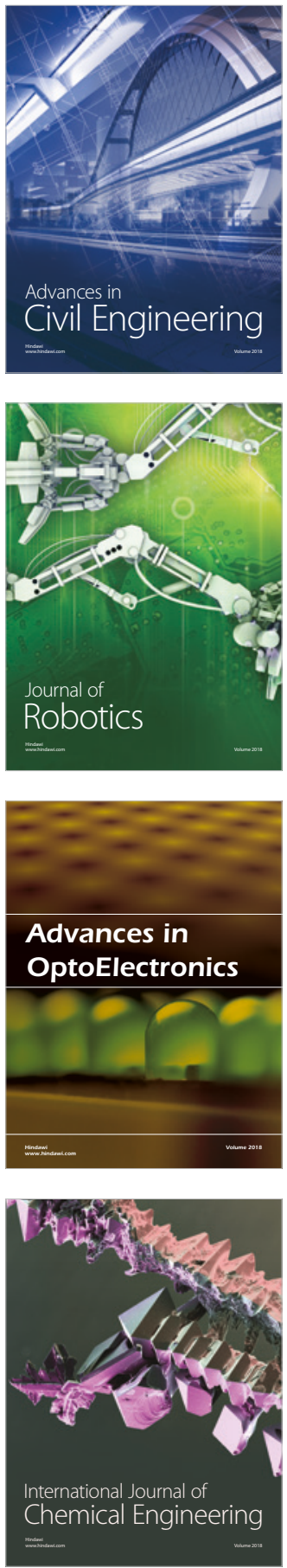

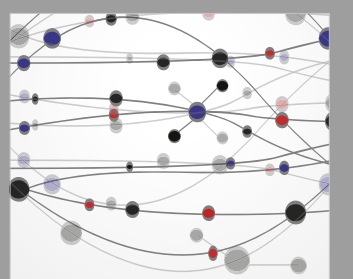

\section{Rotating \\ Machinery}

The Scientific World Journal

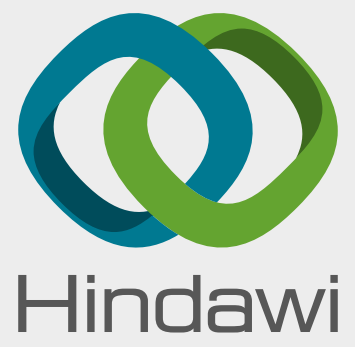

Submit your manuscripts at

www.hindawi.com
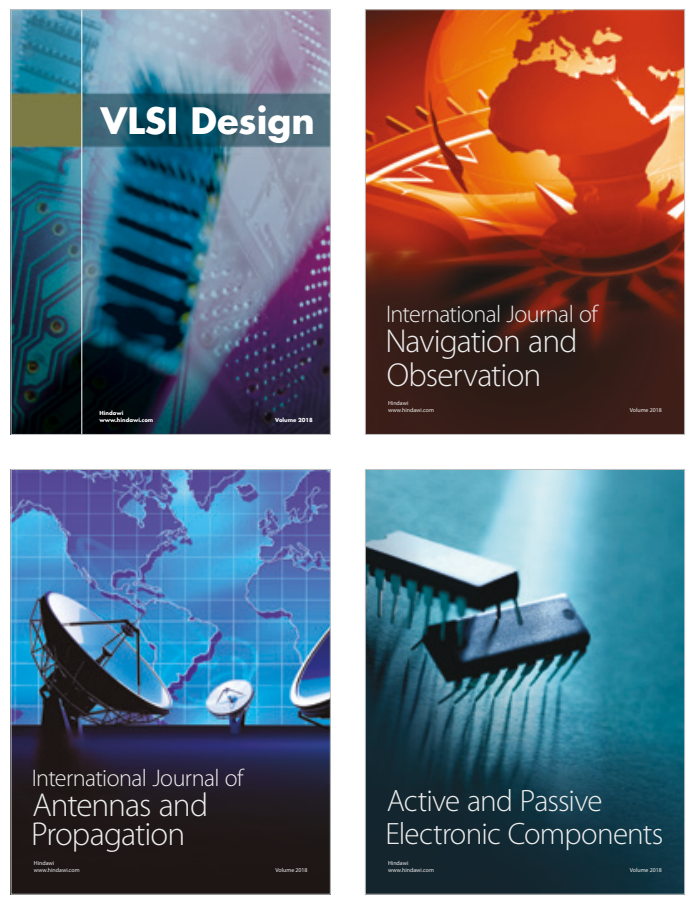
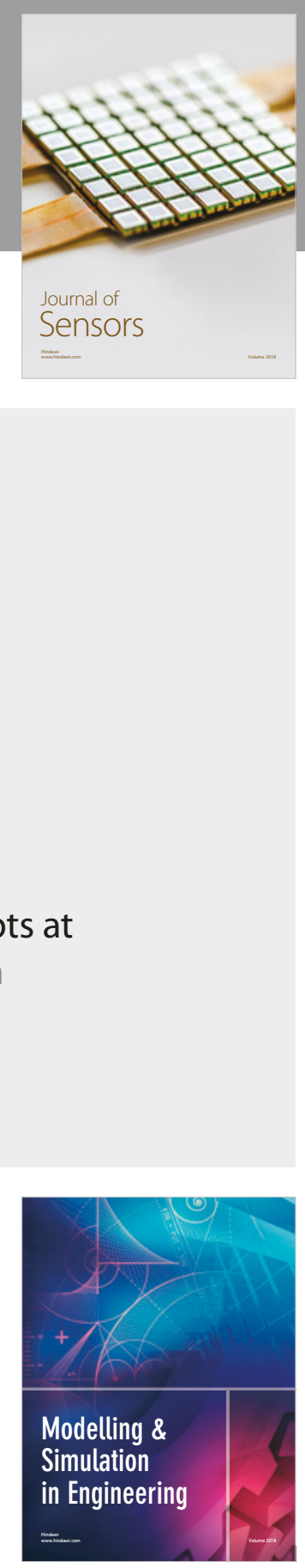

\section{Advances \\ Multimedia}
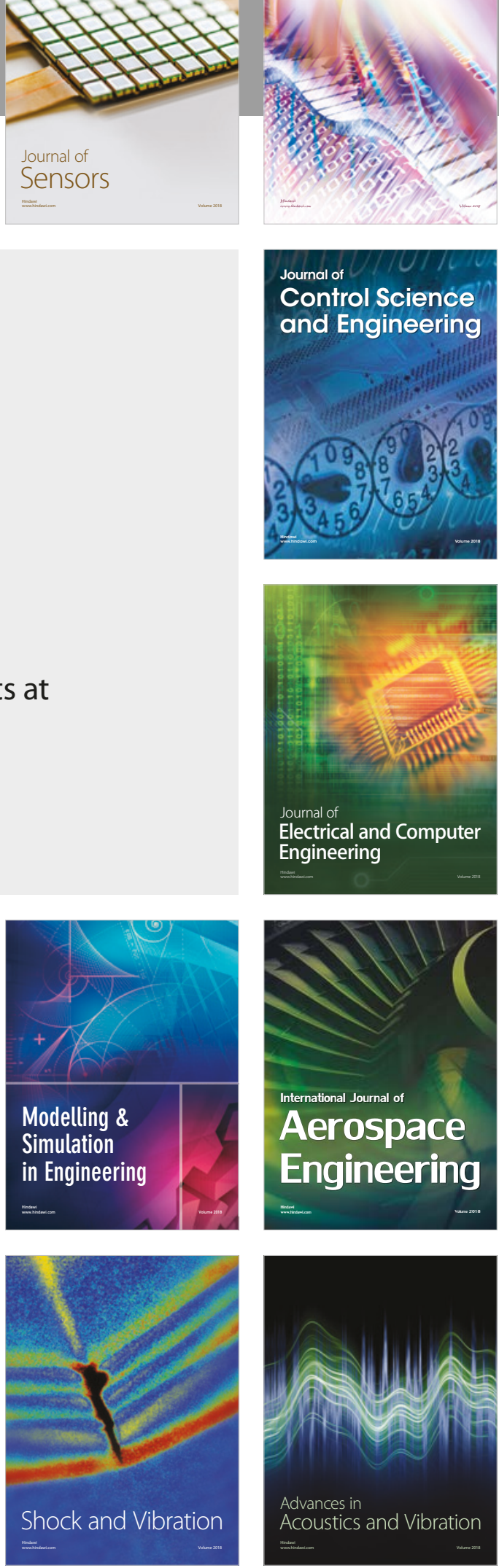